\title{
GMR
}

\section{Genetic diversity and selection of common bean lines based on technological quality and biofortification}

\author{
S. de M. Steckling ${ }^{1}$, N.D. Ribeiro ${ }^{1}$, F.D. Arns ${ }^{1}$, H.C. Mezzomo ${ }^{1}$ and \\ M.T.D.F. Possobom² \\ ${ }^{1}$ Departamento de Fitotecnia, Universidade Federal de Santa Maria, Santa Maria, \\ RS, Brasil \\ ${ }^{2}$ Departamento de Fitotecnia, Universidade Federal de Viçosa, Viçosa, MG, Brasil \\ Corresponding author: N.D. Ribeiro \\ E-mail: nerineia@hotmail.com / nerineia.ribeiro@gmail.com
}

Genet. Mol. Res. 16 (1): gmr16019527

Received November 7, 2016

Accepted December 19, 2016

Published March 22, 2017

DOI http://dx.doi.org/10.4238/gmr16019527

Copyright $(92017$ The Authors. This is an open-access article distributed under the terms of the Creative Commons Attribution ShareAlike (CC BY-SA) 4.0 License.

\begin{abstract}
The development of common bean cultivars with high technological quality that are biofortified with minerals, is required to meet the demand for food with health benefits. The objectives of this study were to evaluate whether common bean genotypes differ in terms of technological and mineral biofortification traits, to study the correlations between these characters, to analyze the genetic dissimilarity of common bean genotypes, and to select superior lines for these traits. For this, 14 common bean genotypes were evaluated in experiments conducted in three growing seasons in the Rio Grande do Sul State, Brazil. A significant genotype $\mathrm{x}$ environment interaction was observed for technological quality (mass of 100 grains and cooking time) and biofortification traits (concentration of potassium, phosphorus, calcium, iron, zinc, and copper). Positive correlation estimates were obtained between phosphorus and potassium $(\mathrm{r}=$ $0.575)$, iron and zinc $(\mathrm{r}=0.641)$, copper and iron $(\mathrm{r}=0.729)$, and
\end{abstract}

Genetics and Molecular Research 16 (1): gmr16019527 
copper and phosphorus $(\mathrm{r}=0.533)$. In the main component cluster analysis, four groups of genotypes were formed. The following lines are recommended for selection: LP 11-363 for fast-cooking, CNFC 11 948 for high iron concentration, and LEC 03-14 for high potassium, phosphorus, and calcium concentrations in grains. Common bean lines with high phosphorus and iron concentrations in grains can be indirectly selected based on higher potassium, copper, and zinc concentrations. Controlled crossings between LP 11-363 x CNFC 11948 and LP 11$363 \times$ LEC 03-14 are recommended to obtain segregating lines that are fast-cooking and biofortified with minerals.

Key words: Phaseolus vulgaris; Cooking time; Minerals; Correlation analysis; Cluster analysis; Selection

\section{INTRODUCTION}

Mineral deficiency in humans can be considered a global public health problem, because it affects people from different countries, different social classes, and of different ages. The most common mineral deficiencies reported in the literature are: iron, which causes anemia and decreases workability (Lynch, 2003); zinc, which affects immune function, growth, and development (Prasad, 2012); and calcium, which causes abnormalities in bone structure, such as osteoporosis (Martínez-Ballesta et al., 2010).

Potassium, phosphorus, and copper deficiencies are not common in humans. However, low potassium intake can lead to increased blood pressure and cardiovascular disease (He and MacGregor, 2008), phosphorus deficiency leads to painful bones, anxiety, and fatigue (Martínez-Ballesta et al., 2010), and reduced copper causes hypochromic anemia, neutropenia, and skeletal disturbances (Guerrero-Romero and Rodríguez-Morán, 2005).

Genetic biofortification is a promising strategy used to prevent mineral deficiencies in humans. Among the crops produced in most countries and consumed by people daily, the common bean (Phaseolus vulgaris L.) is a strategic crop for use in biofortification programs. Common bean contains high concentrations of potassium, phosphorus, calcium, iron, zinc, and copper (Blair et al., 2010; Talukder et al., 2010; Tryphone and Nchimbi-Msolla, 2010; Akond et al., 2011; Jost et al., 2013; Ribeiro et al., 2013a,b, 2014a; Maziero et al., 2015) and is consumed without the need to polish or remove the seed coat, i.e., integrally. In addition, common bean is a source of vegetable protein used widely in the diet of people with low incomes, vegetarians, and vegans. Therefore, it is consumed widely throughout the world by people from different social classes.

Biofortification with minerals has been successfully performed by common bean breeding programs. As a result, common bean lines containing high concentrations of potassium, phosphorus, calcium, iron, zinc, and copper in grains have been obtained (Blair et al., 2010; Jost et al., 2013; Ribeiro et al., 2013a,b, 2014a; Pereira et al., 2014; Maziero et al., 2015). In these lines, the iron concentration exceeds $95 \mathrm{mg} / \mathrm{kg}$ dry matter (DM) (Jost et al., 2013; Ribeiro et al., 2013a), zinc exceeds $31 \mathrm{mg} / \mathrm{kg}$ DM (Tryphone and Nchimbi-Msolla, 2010; Zemolin et al., 2016), and calcium exceeds $1.4 \mathrm{~g} / \mathrm{kg}$ DM (Ribeiro et al., 2013a). For the other minerals, no classes have been defined that characterize the goal of common bean biofortification programs. Furthermore, for the biofortified common bean cultivars can be

Genetics and Molecular Research 16 (1): gmr16019527 
accepted for consumption it is necessary to evaluate whether there is any change in cooking time or in the grain pattern most preferred by the consumer.

Analyses of genetic dissimilarity are important to identify duplication, to select superior lines, and to plan new controlled crossings aimed at common bean biofortification. The application of the analyses of genetic dissimilarity has allowed the identification of groups of common bean lines that differ for two (Blair et al., 2010; Talukder et al., 2010) and four minerals (Pereira et al., 2011). However, studies evaluating the genetic dissimilarity of common bean lines obtained by breeding programs, considering technological quality and biofortification with minerals are lacking in the literature.

Therefore, the objectives of this study were to evaluate whether common bean genotypes differ in terms of technological quality and biofortification with mineral traits, to study the correlations between these characters, to analyze the genetic dissimilarity of common bean genotypes, and to select superior common bean lines for these traits.

\section{MATERIAL AND METHODS}

\section{Growth conditions}

The experiments were conducted in the field area of the Common Bean Breeding Program of the Federal University of Santa Maria, Santa Maria, Rio Grande do Sul (RS), Brazil, in three growing seasons: 2014 rainy, 2015 dry, and 2015 rainy. The rainy (sowing between the months of September to November) and dry (sowing in January and February) growing seasons are traditional in southern Brazil.

Santa Maria is located at the latitude $29^{\circ} 42^{\prime} \mathrm{S}$, longitude $53^{\circ} 49^{\prime} \mathrm{W}$, and $95 \mathrm{~m}$ above sea level (asl). The climate is humid subtropical, with rainfall usually well distributed throughout the year, according to the Köppen classification. The soil of the experimental area is a typical alitic Argisol (Hapludalf), and was prepared in the conventional manner. The amount of fertilizer needed for the experiments was calculated based on the interpretation of the soil chemical analysis report. Fertilizer applied at the base was $200 \mathrm{~kg} / \mathrm{ha}$ of the 5-20-20 formula (urea: $45 \%$ nitrogen, superphosphate: $18 \% \mathrm{P}_{2} \mathrm{O}_{5}$, and potassium chloride: $60 \% \mathrm{~K}_{2} \mathrm{O}$ ). In furrow sowing, $50 \mathrm{~kg} / \mathrm{ha}$ nitrogen was applied in the form of urea at the first trifoliate leaf stage (V3).

The experimental design was a randomized block, with four replicates. Treatments consisted of 14 common bean genotypes evaluated in the South Brazilian Use and Cultivation Value experiment. Of these, 10 lines were obtained by different breeders: CHC 01-175-1, CHP 99.6524 (Empresa de Pesquisa Agropecuária e Extensão Rural de Santa Catarina - EPAGRI, Santa Catarina), CNFC 11 954, CNFC 11948 (Empresa Brasileira de Pesquisa Agropecuária - EMBRAPA Arroz e Feijão, Goiás), LP 12-601, LP 11-363 (Instituto Agronômico do Paraná - IAPAR, Paraná), FAP F3-2, C10-2-4/41 (Instituto Agronômico de Campinas - IAC, São Paulo), and LEP 04-14, LEC 03-14 (Universidade Estadual de Maringá - UEM, Paraná), and four cultivars were used as controls (Pérola, BRS Valente, Guapo Brilhante, and Carioca). The inbred lines represent the technological advances of common bean breeding in public institutions of southern Brazil in the biennium 2014 and 2015. All genotypes evaluated belong to the Mesoamerican gene pool and characterize the most consumed bean types in Brazil: Black (LP 12-601, CHP 99.65 24, LEP 04-14, BRS Valente, and Guapo Brilhante) and Carioca (other lines and cultivars). The experimental unit consisted of four $4.0-\mathrm{m}$ long rows, $0.50-\mathrm{m}$ apart. The two central rows of each experimental unit were considered as the useful area $\left(4 \mathrm{~m}^{2}\right)$.

Genetics and Molecular Research 16 (1): gmr16019527 
Sowing was performed manually, with 16 seeds per linear meter for the indeterminate growth habit genotypes (IGH) with short guides (type II), and 13 seeds per linear meter for the IGH genotypes with long guides (type III). Seeds were treated with Maxim XL fungicide (Fludioxonil and Metalaxyl-M) and the Cruiser insecticide (Thiamethoxam), both at a dose of $200 \mathrm{~mL} / 100 \mathrm{~kg}$ seeds.

During the vegetative development of common bean plants, insect control was carried out with the application of Engeo ${ }^{\mathrm{TM}}$ (Thiamethoxam and lambdacyhalothrin) at a dose of 125 $\mathrm{mL} / \mathrm{ha}$. The weeds were eliminated by hoeing. No fungicide was applied for disease control.

\section{Technological quality and biofortification traits}

Common bean plants within the useful area were harvested and threshed by hand at the maturity stage to prevent metal contamination. Broken grains and impurities were removed and grains were dried in a forced-air oven $\left(65^{\circ} \mathrm{C}\right)(1.5$ Odontobrás, Odontobrás, Ribeirão Preto, SP, Brazil) at $13 \%$ average moisture. The mass of 100 grains was determined by counting and weighing three samples of 100 grains from each genotype, in a balance with precision of the two decimal.

The grains were placed in a plastic cup containing $50 \mathrm{~mL}$ distilled water and soaked for $8 \mathrm{~h}$ at room temperature $\left(20^{\circ} \pm 2^{\circ} \mathrm{C}\right)$. Cooking time was determined using a Mattson cooker of 25 pegs (EMBRAPA Instrumentation, São Carlos, SP, Brazil) according to the methodology described by Ribeiro et al. (2014a). The mean falling time of the first 13 pegs was considered as the cooking time of each sample (Ribeiro et al., 2007).

Samples of grains (50 g) were ground in a knife micro-mill (Q298A21, Quimis, São Paulo, SP, Brazil) to obtain particles smaller than $1 \mathrm{~mm}$ in diameter, and were not sieved. The mineral concentration was determined in aliquots containing $0.5 \mathrm{~g}$ of the raw bean flour obtained by nitric-perchloric digestion, according to the method described by Jost et al. (2013). The potassium concentration was read using a flame photometer (B262, Micronal, São Paulo, SP, Brazil) and the phosphorus concentration was measured in an optical emission spectrophotometer (AA-7000, Shimadzu, São Paulo, SP, Brazil). The concentrations of calcium, iron, zinc, and copper were determined using an atomic absorption spectrophotometer (ICE 3000, Thermo Scientific, San Jose, USA), with wavelengths of 422.70, 248.30, 213.90, and $324.8 \mathrm{~nm}$, respectively.

\section{Statistical analysis}

The data were submitted to individual and joint analyses of variance. The homogeneity of residual variance was tested using Hartley's Fmax test (Cruz et al., 2012). The F test (P < 0.05 ) was used to test the hypotheses of the main effects (genotype and environment) and the genotype $\mathrm{x}$ environment interaction $(\mathrm{G} \times \mathrm{E})$, considering these effects as fixed. The ScottKnott test at 0.05 probability was used to group genotypes and environments.

Pearson linear correlation coefficients were estimated using the phenotypic correlation matrix among traits. The significance of the correlation coefficients was tested by the Student's $t$ test $(\mathrm{P}<0.05)$. The multicolinearity diagnostic was accomplished using the phenotypic correlation matrix, and its effect evaluated by the number of conditions, as defined by Montgomery and Peck (1981).

The mean data obtained in the three environments were used for the genetic

Genetics and Molecular Research 16 (1): gmr16019527 
dissimilarity analysis. Principal components analysis was performed to identify the traits with greater and lesser contribution to the total variation and cluster analysis. For this purpose, a dispersion graph was generated from the first two principal components. The first principal component was represented by the $\mathrm{x}$-axis and the second principal component by the y-axis, allowing visualization of the groups formed. All statistical analyses were performed using Microsoft Office Excel and Genes software (Cruz, 2016).

\section{RESULTS AND DISCUSSION}

\section{Joint analysis of variance}

The variance of the experimental error was homogeneous for all traits evaluated in three environments $(P>0.05)$, enabling a joint analysis of variance to be performed. In the joint analysis of variance, a significant $\mathrm{G} x \mathrm{E}$ interaction was observed for all traits (Table 1). As a result, the technological quality traits and mineral concentration in grains of common bean genotypes were not constant under different growing environments. The differential response of common bean genotypes to growing environments has been previously described for the mass of 100 grains, cooking time (Ribeiro et al., 2013a, 2014b), and concentrations of potassium, phosphorus, calcium, iron, zinc, and copper in grains (Hossain et al., 2013; Pereira et al., 2014). Thus, there is genetic variability for the evaluated traits, which enables the selection of common bean lines with high technological quality traits that are biofortified with minerals.

Table 1. Joint analysis of variance containing the mean squares, degrees of freedom (DF), average, variation coefficient (VC\%) for the mass of 100 grains (M100G, g), cooking time (cooking, second), concentrations of potassium $(\mathrm{K}, \mathrm{g} / \mathrm{kg}$ of dry matter $[\mathrm{DM}])$, phosphorus $(\mathrm{P}, \mathrm{g} / \mathrm{kg} \mathrm{DM})$, calcium $(\mathrm{Ca}, \mathrm{g} / \mathrm{kg} \mathrm{DM})$, iron $(\mathrm{Fe}, \mathrm{mg} / \mathrm{kg}$ $\mathrm{DM})$, zinc $(\mathrm{Zn}, \mathrm{mg} / \mathrm{kg} \mathrm{DM})$, and copper $(\mathrm{Cu}, \mathrm{mg} / \mathrm{kg} \mathrm{DM})$ in grains of 14 common bean genotypes evaluated in three experiments carried out in 2014 and 2015.

\begin{tabular}{|c|c|c|c|c|c|c|c|c|c|}
\hline \multirow[t]{2}{*}{ Sources of variation } & \multicolumn{9}{|c|}{ Mean square } \\
\hline & d.f. & M100G & Cooking & $\mathrm{K}$ & $\mathrm{P}$ & $\mathrm{Ca}$ & $\mathrm{Fe}$ & $\mathrm{Zn}$ & $\mathrm{Cu}$ \\
\hline Block/Environment & 6 & 3.02 & $11,485.14$ & 0.57 & 0.58 & 0.21 & 142.14 & 2.57 & 0.86 \\
\hline Genotype (G) & 13 & $19.71 *$ & $64,294.45^{*}$ & $1.29^{*}$ & $0.64 *$ & $1.55^{*}$ & $862.04 *$ & $15.96^{*}$ & $4.40^{*}$ \\
\hline Environment (E) & 2 & $4.26^{\mathrm{ns}}$ & $2,207,895.72 *$ & $15.10^{*}$ & $8.56^{*}$ & $23.55^{*}$ & $10,972.44 *$ & $40.95 *$ & $186.40^{*}$ \\
\hline $\mathrm{G} \times \mathrm{E}$ & 26 & $7.02 *$ & $16,970.54 *$ & $2.02 *$ & $0.34 *$ & $0.61 *$ & $614.98^{*}$ & $10.32 *$ & $1.38^{*}$ \\
\hline Residue & 78 & 1.91 & $7,793.99$ & 0.54 & 0.18 & 0.13 & 63.96 & 3.36 & 0.44 \\
\hline Mean & & 22.15 & $1,009.75$ & 12.30 & 4.93 & 3.46 & 89.01 & 22.95 & 8.36 \\
\hline $\mathrm{VC}(\%)$ & & 6.24 & 8.74 & 6.00 & 8.67 & 10.46 & 8.98 & 7.99 & 7.95 \\
\hline
\end{tabular}

*Significant by the $\mathrm{F}$ test $(\mathrm{P}=0.05)$; $\mathrm{ns}=$ non-significant.

\section{Technological quality traits}

The mass of 100 grains of common bean genotypes varied from 17.21 (LP 12-601, 2015 rainy season) to 26.76 g (Pérola, 2014 rainy season) (Table 2), and was within the expected range for common bean grains from the Mesoamerican gene pool (Blair et al., 2010). None of the genotypes evaluated showed a medium size for mass of 100 grains ( 25 to $30 \mathrm{~g}$ ) for the three environments. This grain pattern is most preferred by packing companies, and by black and Carioca-type common bean consumers in Brazil, according to Carbonell et al. (2010). Therefore, this pattern has been prioritized in the selection of common bean lines. In

Genetics and Molecular Research 16 (1): gmr16019527 
the present study, the high rainfall recorded during the three growing seasons (Table 3) may have affected grain filling, contributing to the mass of 100 grains being lower than expected for the different genotypes. The mass of 100 grains in common bean is greatly influenced by the environment (Ribeiro et al., 2013a, 2014b; dos Santos et al., 2016).

Table 2. Average* mass of 100 grains, cooking time (min: s), potassium and phosphorus concentrations in grains of 14 common bean genotypes evaluated in three experiments carried out in 2014 and 2015.

\begin{tabular}{|c|c|c|c|c|c|c|c|c|c|c|c|c|}
\hline \multirow{3}{*}{$\begin{array}{l}\text { Genotype } \\
\text { Pérola }\end{array}$} & \multicolumn{6}{|c|}{ Mass of 100 grains $(\mathrm{g})$} & \multicolumn{6}{|c|}{ Cooking time (min:s) } \\
\hline & \multicolumn{2}{|c|}{ Env. $1^{1}$} & \multicolumn{2}{|c|}{ Env. 2} & \multicolumn{2}{|c|}{ Env. 3} & \multicolumn{2}{|c|}{ Env. 1} & \multicolumn{2}{|c|}{ Env. 2} & \multicolumn{2}{|c|}{ Env. 3} \\
\hline & 26.76 & $\mathrm{aA}$ & 22.88 & a B & 20.90 & $\mathrm{cB}$ & $16: 00$ & a B & $13: 33$ & a B & $22: 58$ & $\mathrm{aA}$ \\
\hline FAP F3-2 & 21.62 & c A & 22.78 & $\mathrm{aA}$ & 20.32 & c A & $17: 52$ & a B & $17: 33$ & a B & $22: 01$ & a A \\
\hline CHC 01-175-1 & 23.46 & $\mathrm{~b} \mathrm{~A}$ & 22.57 & $\mathrm{aA}$ & 21.59 & c A & $16: 40$ & a B & $14: 32$ & $\mathrm{aC}$ & $24: 02$ & a A \\
\hline LP 12-601 & 20.24 & $\mathrm{c} \mathrm{A}$ & 21.64 & a A & 17.21 & d B & $14: 39$ & b B & $14: 12$ & a B & $20: 22$ & $\mathrm{bA}$ \\
\hline BRS Valente & 20.08 & $\mathrm{c} \mathrm{A}$ & 21.76 & a A & 21.13 & c A & $17: 34$ & a B & $12: 39$ & $\mathrm{aC}$ & $20: 43$ & $\mathrm{bA}$ \\
\hline LP 11-363 & 22.66 & $\mathrm{~b} \mathrm{~A}$ & 21.22 & a A & 21.67 & c A & $12: 43$ & $\mathrm{bB}$ & $11: 43$ & a B & $15: 37$ & $\mathrm{c} \mathrm{A}$ \\
\hline CHP 99.6524 & 20.29 & $\mathrm{c} \mathrm{A}$ & 21.23 & a A & 22.67 & $\mathrm{~b} \mathrm{~A}$ & $14: 04$ & $\mathrm{bB}$ & $13: 15$ & a B & 19:27 & $\mathrm{bA}$ \\
\hline LEP 04-14 & 21.55 & c B & 23.11 & a B & 25.87 & a A & $14: 08$ & $\mathrm{bB}$ & $13: 52$ & a B & $22: 33$ & $\mathrm{aA}$ \\
\hline G. Brilhante & 20.33 & c A & 20.42 & a A & 17.87 & d B & $15: 35$ & b B & $13: 25$ & a B & $23: 02$ & a A \\
\hline CNFC 11954 & 23.72 & $\mathrm{~b} \mathrm{~A}$ & 23.09 & a A & 23.60 & $\mathrm{~b} \mathrm{~A}$ & 18:03 & a B & $13: 38$ & $\mathrm{aC}$ & $22: 21$ & $\mathrm{aA}$ \\
\hline CNFC 11948 & 23.26 & b A & 23.05 & a A & 25.33 & a A & $17: 15$ & a B & 13:09 & $\mathrm{aC}$ & $21: 59$ & a A \\
\hline C10-2-4/41 & 22.76 & $\mathrm{~b} \mathrm{~A}$ & 25.64 & $\mathrm{aA}$ & 24.27 & a A & $13: 32$ & $\mathrm{bB}$ & 14:01 & $\mathrm{aB}$ & $20: 10$ & $\mathrm{~b} \mathrm{~A}$ \\
\hline Carioca & 21.08 & $\mathrm{c} \mathrm{A}$ & 23.73 & a A & 22.40 & $\mathrm{~b} \mathrm{~A}$ & $16: 50$ & a B & $14: 20$ & $\mathrm{aC}$ & $21: 03$ & $\mathrm{~b} \mathrm{~A}$ \\
\hline LEC 03-14 & 22.12 & $\mathrm{c} \mathrm{A}$ & 21.56 & $\mathrm{aA}$ & 20.93 & $\mathrm{c} \mathrm{A}$ & $16: 26$ & $\mathrm{aB}$ & $14: 15$ & $\mathrm{aC}$ & $22: 02$ & a A \\
\hline Mean & 22.14 & & 22.48 & & 21.84 & & $15: 49$ & & $13: 52$ & & $21: 30$ & \\
\hline VC (\%) & 7.07 & & 4.07 & & 7.17 & & 7.64 & & 9.80 & & 8.55 & \\
\hline \multirow[t]{2}{*}{ Genotype } & \multicolumn{6}{|c|}{ Potassium ( $\mathrm{g} / \mathrm{kg}$ dry matter) } & \multicolumn{6}{|c|}{ Phosphorus ( $\mathrm{g} / \mathrm{kg}$ dry matter) } \\
\hline & \multicolumn{2}{|c|}{ Env. 1} & \multicolumn{2}{|c|}{ Env. 2} & \multicolumn{2}{|c|}{ Env. 3} & \multicolumn{2}{|c|}{ Env. 1} & \multicolumn{2}{|c|}{ Env. 2} & \multicolumn{2}{|c|}{ Env. 3} \\
\hline Pérola & 12.17 & a A & 11.51 & $\mathrm{aA}$ & 12.83 & $\mathrm{aA}$ & 5.48 & $\mathrm{aA}$ & 4.35 & a B & 5.19 & $\mathrm{aA}$ \\
\hline FAP F3-2 & 12.76 & a A & 12.25 & a A & 13.42 & a A & 5.42 & a A & 4.64 & a B & 4.52 & b B \\
\hline CHC 01-175-1 & 12.39 & $\mathrm{aA}$ & 12.83 & $\mathrm{aA}$ & 12.32 & $\mathrm{aA}$ & 5.08 & $\mathrm{bA}$ & 4.73 & $\mathrm{aA}$ & 4.78 & $\mathrm{bA}$ \\
\hline LP 12-601 & 13.42 & a A & 12.98 & $\mathrm{aA}$ & 10.12 & $\mathrm{c} \mathrm{B}$ & 5.64 & a A & 4.29 & $\mathrm{aB}$ & 4.47 & $\mathrm{bB}$ \\
\hline BRS Valente & 12.54 & $\mathrm{aA}$ & 12.32 & $\mathrm{aA}$ & 9.97 & $\mathrm{c} \mathrm{B}$ & 4.58 & $\mathrm{bA}$ & 4.62 & $\mathrm{aA}$ & 4.46 & $\mathrm{bA}$ \\
\hline LP 11-363 & 12.69 & a A & 12.76 & a A & 11.15 & b B & 5.58 & $\mathrm{aA}$ & 4.67 & a B & 4.83 & $\mathrm{bB}$ \\
\hline CHP 99.6524 & 13.86 & a A & 11.81 & a B & 11.81 & b B & 6.24 & $\mathrm{aA}$ & 4.39 & $\mathrm{aC}$ & 5.40 & $\mathrm{aB}$ \\
\hline LEP 04-14 & 13.49 & a A & 12.39 & a B & 11.66 & b B & 4.79 & $\mathrm{~b} \mathrm{~A}$ & 4.59 & a A & 4.65 & $\mathrm{~b} \mathrm{~A}$ \\
\hline G. Brilhante & 13.27 & a A & 13.57 & a A & 11.22 & b B & 6.10 & a A & 4.62 & a B & 5.08 & a B \\
\hline CNFC 11954 & 12.83 & $\mathrm{aA}$ & 11.95 & a A & 11.22 & b A & 4.85 & $\mathrm{~b} A$ & 4.89 & a B & 4.72 & $\mathrm{~b} \mathrm{~A}$ \\
\hline CNFC 11948 & 12.25 & a A & 11.95 & a A & 11.37 & b A & 5.09 & $\mathrm{~b} A$ & 4.53 & a B & 5.48 & $\mathrm{a} \mathrm{A}$ \\
\hline C10-2-4/41 & 13.49 & a A & 11.37 & a B & 12.83 & a A & 5.72 & a A & 4.37 & a B & 5.37 & a A \\
\hline Carioca & 12.69 & $\mathrm{aA}$ & 11.59 & $\mathrm{aB}$ & 11.29 & b B & 5.39 & a A & 4.28 & $\mathrm{aB}$ & 4.43 & $\mathrm{bB}$ \\
\hline LEC 03-14 & 13.20 & a A & 12.10 & a A & 13.13 & $\mathrm{aA}$ & 5.62 & $\mathrm{aA}$ & 5.02 & a A & 5.02 & $\mathrm{aA}$ \\
\hline Mean & 12.93 & & 12.24 & & 11.74 & & 5.40 & & 4.50 & & 4.88 & \\
\hline $\mathrm{VC}(\%)$ & 4.47 & & 7.24 & & 6.11 & & 10.07 & & 8.40 & & 6.74 & \\
\hline
\end{tabular}

*Means not followed by the same lower case letter in the column differ by the Scott-Knott test $(\mathrm{P}=0.05)$, and uppercase letters on each row by the Scott-Knott test $(\mathrm{P}=0.05) .{ }^{1}$ Environments: Env. 1: 2014 rainy season; Env. 2: 2015 dry season; Env. 3: 2015 rainy season.

Common bean lines had a cooking time equal to or less than that of the Pérola, BRS Valente, Guapo Brilhante, and Carioca cultivars (Table 2). All genotypes evaluated presented a cooking time of less than $25 \mathrm{~min}$, which is characteristic of fast-cooking, according to dos Santos et al. (2016). However, cooking time varied from $11 \mathrm{~min} 43 \mathrm{~s}$ to $15 \mathrm{~min} 37 \mathrm{~s}$ for the LP 11-363 line, in the three environments evaluated. The development of very fast-cooking common bean cultivars, i.e., those that reach softness considered ideal for consuming before less than 15 min cooking, meets the demand for fast preparation food. Common bean lines that cook in less than 15 min were previously identified by Ribeiro et al. (2013 a,b). 
Table 3. Meteorological data showing average monthly minimum temperature (Tmin), maximum temperature (Tmax), mean temperature (Tmed), and rainfall (Rain) during the 2014 rainy, 2015 dry, and 2015 rainy seasons collected in the 8th Meteorology District, in Santa Maria Meteorological Station, set at the Federal University of Santa Maria $\left(29^{\circ} 42^{\prime} \mathrm{S}, 53^{\circ} 49^{\prime} \mathrm{W}\right.$, and $95 \mathrm{~m}$ asl), in the State of Rio Grande do Sul, Brazil.

\begin{tabular}{l|c|c|c|c|c|c|c|c|c|c|c|c|c|c}
\hline & \multicolumn{9}{|c|}{ Rainy season 2014 } & \multicolumn{4}{c|}{ Dry season 2015 } & \multicolumn{4}{c}{ Rainy season 2015 } \\
\hline & Oct & Nov & Dec & Jan & Feb & Mar & Apr & May & Jun & Jul & Nov & Dec & Jan & Feb \\
\hline$T \min \left({ }^{\circ} \mathrm{C}\right)$ & 17.0 & 17.4 & 19.3 & 20.8 & 20.2 & 18.8 & 15.1 & 13.1 & 10.7 & 11.5 & 16.9 & 19.6 & 20.8 & 21.2 \\
\hline $\mathrm{T} \max \left({ }^{\circ} \mathrm{C}\right)$ & 27.0 & 29.7 & 29.7 & 30.8 & 30.0 & 29.9 & 27.0 & 22.4 & 20.9 & 19.4 & 25.5 & 28.6 & 31.5 & 31.3 \\
\hline $\mathrm{T} \operatorname{med}\left({ }^{\circ} \mathrm{C}\right)$ & 22.0 & 23.5 & 24.5 & 25.8 & 25.1 & 24.3 & 21.0 & 17.7 & 15.8 & 15.4 & 21.2 & 24.1 & 26.1 & 26.2 \\
\hline Rain $(\mathrm{mm})$ & 256.8 & 59.7 & 198.1 & 54.9 & 84.3 & 132.4 & 129.8 & 136.4 & 128.2 & 214.7 & 164.3 & 336.0 & 101.9 & 96.5 \\
\hline
\end{tabular}

\section{Biofortification traits}

In this study, a significant difference was found for potassium concentration in grains by genotype via the F test (Table 1). However, no stratification among treatments was observed at the same significance level in the 2014 rainy and 2015 dry seasons by Scott-Knott test (Table 2). In the 2015 rainy season, three groups of genotypes were formed. The group with the lowest potassium values (less than $10.12 \mathrm{~g} / \mathrm{kg}$ DM) contained the LP 12-601 line and the BRS Valente cultivar. The FAP F3-2, CHC 01-175-1, C10-2-4/41, and LEC 03-14 lines and the Pérola cultivar formed the group with the highest potassium values (from 12.32 to 13.42 $\mathrm{g} / \mathrm{kg} \mathrm{DM}$ ). These potassium values were similar to those obtained in black and Carioca-type common bean genotypes (Ribeiro et al., 2013b; Maziero et al., 2015), and lower than those observed in common bean of varied colors (Pinheiro et al., 2010; Pereira et al., 2011; Silva et al., 2012; Ribeiro et al., 2014a).

Based on the results of this study, the following classification is proposed for potassium concentration in common bean grains: low $(<10 \mathrm{~g} / \mathrm{kg} \mathrm{DM})$ and high $(>12 \mathrm{~g} / \mathrm{kg} \mathrm{DM})$. The FAP F3-2, CHC 01-175-1, C10-2-4/41, and LEC 03-14 lines, and the Pérola cultivar have a high potassium concentration in their grains. Common bean biofortified with potassium is important for the control of high blood pressure and cardiovascular disease, which, according to He and MacGregor (2008), are caused by low dietary potassium intake.

Common bean genotypes were classified into two groups based on their phosphorus concentrations in the 2014 rainy season: group 1 (4.58 to $5.09 \mathrm{~g} / \mathrm{kg} \mathrm{DM}$ ) and group 2 (5.39 to $6.24 \mathrm{~g} / \mathrm{kg} \mathrm{DM}$ ). In the 2015 rainy season, the range was from 4.43 to $4.83 \mathrm{~g} / \mathrm{kg} \mathrm{DM}$ in group 1 and from 5.02 to $5.48 \mathrm{~g} / \mathrm{kg} \mathrm{DM}$ in group 2. Those phosphorus values are within the range found in common bean genotypes grown in the United States (Hacisalihoglu and Settles, 2013; Hossain et al., 2013), Portugal (Pinheiro et al., 2010), and Brazil (Pereira et al., 2011; Silva et al., 2012; Maziero et al., 2015). Therefore, common bean lines with a phosphorus concentration higher than $5.00 \mathrm{~g} / \mathrm{kg}$ DM should be considered of high nutritional value due to the phosphorus values in grains.

In the present study, the LEC 03-14 line was identified as having a high phosphorus concentration under the three environments investigated. Similarly, Maziero et al. (2015) selected common bean lines with high phosphorus concentration in their grains, independently of the growing environment. This shows that common bean can be biofortified with phosphorus and used to reduce symptoms such as painful bones, anxiety, fatigue, and skin sensitivity, which are observed in cases of phosphorus deficiency in humans (Martínez-Ballesta et al., 2010).

The lowest calcium value was observed for the C10-2-4/41 line (1.85 g/kg DM, 2015 dry season) and the highest value was observed for the CNFC 11954 line $(4.81 \mathrm{~g} / \mathrm{kg} \mathrm{DM}$,

Genetics and Molecular Research 16 (1): gmr16019527 
2014 rainy season) (Table 4). These calcium values are higher than those previously described for common bean grains (Pinheiro et al., 2010; Akond et al., 2011; Pereira et al., 2011; Jost et al., 2013; Ribeiro et al., 2013a,b; Morais et al., 2016) and characterize a high calcium concentration (Ribeiro et al., 2013a).

Table 4. Average* calcium, iron, zinc, and copper concentrations in grains of 14 common bean genotypes evaluated in three experiments carried out in 2014 and 2015.

\begin{tabular}{|c|c|c|c|c|c|c|c|c|c|c|c|c|}
\hline \multirow{3}{*}{$\begin{array}{l}\text { Genotype } \\
\text { Pérola }\end{array}$} & \multicolumn{6}{|c|}{ Calcium (g/kg dry matter) } & \multicolumn{6}{|c|}{ Iron (mg/kg dry matter) } \\
\hline & \multicolumn{2}{|c|}{ Env. 1} & \multicolumn{2}{|c|}{ Env. 2} & \multicolumn{2}{|c|}{ Env. 3} & \multicolumn{2}{|c|}{ Env. 1} & \multicolumn{2}{|c|}{ Env. 2} & \multicolumn{2}{|c|}{ Env. 3} \\
\hline & 3.86 & $\mathrm{~b} \mathrm{~A}$ & 3.23 & $\mathrm{aA}$ & 3.37 & $\mathrm{c} \mathrm{A}$ & 85.27 & $\mathrm{~d} \mathrm{~A}$ & 77.00 & $\mathrm{c} \mathrm{A}$ & 76.03 & $\mathrm{~b} \mathrm{~A}$ \\
\hline FAP F3-2 & 3.51 & $\mathrm{~b} \mathrm{~A}$ & 3.13 & a A & 2.31 & $\mathrm{~dB}$ & 78.38 & $\mathrm{~d} \mathrm{~A}$ & 79.62 & $\mathrm{~b} \mathrm{~A}$ & 69.17 & $\mathrm{~b} \mathrm{~A}$ \\
\hline CHC 01-175-1 & 4.20 & $\mathrm{aA}$ & 2.30 & $\mathrm{bB}$ & 4.29 & $\mathrm{aA}$ & 140.68 & $\mathrm{aA}$ & 72.86 & $\mathrm{cB}$ & 67.67 & $\mathrm{bB}$ \\
\hline LP 12-601 & 3.58 & $\mathrm{~b} \mathrm{~A}$ & 2.72 & a B & 3.59 & $\mathrm{~b} \mathrm{~A}$ & 103.61 & $\mathrm{c} \mathrm{A}$ & 70.08 & $\mathrm{c} \mathrm{B}$ & 72.57 & b B \\
\hline BRS Valente & 4.38 & $\mathrm{aA}$ & 2.14 & $\mathrm{~b} \mathrm{~B}$ & 4.17 & $\mathrm{aA}$ & 74.34 & $\mathrm{dA}$ & 80.84 & $\mathrm{~b} \mathrm{~A}$ & 70.40 & $\mathrm{~b} \mathrm{~A}$ \\
\hline LP 11-363 & 3.59 & b A & 2.22 & $\mathrm{bB}$ & 3.34 & c A & 101.00 & c A & 79.63 & b B & 92.17 & $\mathrm{aA}$ \\
\hline CHP 99.6524 & 4.64 & a A & 2.62 & $\mathrm{aC}$ & 3.81 & b B & 138.83 & $\mathrm{aA}$ & 70.40 & $\mathrm{cC}$ & 94.87 & a B \\
\hline LEP 04-14 & 4.16 & a A & 2.63 & $\mathrm{aC}$ & 3.39 & $\mathrm{c} \mathrm{B}$ & 134.31 & $\mathrm{aA}$ & 66.19 & $\mathrm{cC}$ & 99.77 & $\mathrm{aB}$ \\
\hline G. Brilhante & 4.72 & a A & 2.77 & $\mathrm{aC}$ & 3.93 & $\mathrm{bB}$ & 120.12 & $\mathrm{~b} \mathrm{~A}$ & 89.57 & $\mathrm{aB}$ & 88.67 & a B \\
\hline CNFC 11954 & 4.81 & a A & 3.29 & $\mathrm{aC}$ & 3.92 & b B & 84.48 & $\mathrm{~d} \mathrm{~A}$ & 64.76 & c B & 91.70 & a A \\
\hline CNFC 11948 & 4.09 & $\mathrm{aA}$ & 2.08 & $\mathrm{bC}$ & 2.87 & $\mathrm{~dB}$ & 113.38 & $\mathrm{~b} \mathrm{~A}$ & 95.00 & a B & 99.47 & a B \\
\hline C10-2-4/41 & 3.57 & b A & 1.85 & $\mathrm{bC}$ & 2.57 & $\mathrm{~dB}$ & 94.24 & c A & 63.00 & c B & 89.07 & a A \\
\hline Carioca & 4.30 & $\mathrm{a} A$ & 3.15 & $\mathrm{aB}$ & 4.53 & $\mathrm{aA}$ & 115.28 & $\mathrm{~b} \mathrm{~A}$ & 73.59 & $\mathrm{cB}$ & 79.93 & $\mathrm{bB}$ \\
\hline LEC 03-14 & 4.10 & $\mathrm{aB}$ & 2.83 & $\mathrm{aC}$ & 4.74 & $\mathrm{a} A$ & 113.36 & b A & 75.79 & $\mathrm{c} \mathrm{C}$ & 91.57 & $\mathrm{aB}$ \\
\hline Mean & 4.11 & & 2.64 & & 3.63 & & 106.95 & & 75.58 & & 84.50 & \\
\hline VC (\%) & 10.00 & & 10.52 & & 10.55 & & 7.52 & & 11.00 & & 9.02 & \\
\hline \multirow[t]{2}{*}{ Genotype } & \multicolumn{6}{|c|}{ Zinc $(\mathrm{g} / \mathrm{kg}$ dry matter) } & \multicolumn{6}{|c|}{ Copper (g/kg dry matter) } \\
\hline & \multicolumn{2}{|c|}{ Env. 1} & \multicolumn{2}{|c|}{ Env. 2} & \multicolumn{2}{|c|}{ Env. 3} & \multicolumn{2}{|c|}{ Env. 1} & \multicolumn{2}{|c|}{ Env. 2} & \multicolumn{2}{|c|}{ Env. 3} \\
\hline Pérola & 20.42 & $\mathrm{~b} \mathrm{~A}$ & 20.51 & $\mathrm{aA}$ & 22.16 & $\mathrm{bA}$ & 8.40 & $\mathrm{c} \mathrm{A}$ & 9.00 & $\mathrm{~b} \mathrm{~A}$ & 6.48 & a B \\
\hline FAP F3-2 & 18.77 & b B & 21.43 & a B & 24.16 & a A & 8.91 & c A & 8.98 & b A & 5.40 & a B \\
\hline CHC 01-175-1 & 21.17 & b A & 21.60 & $\mathrm{aA}$ & 21.35 & b A & 9.11 & c A & 11.40 & $\mathrm{aA}$ & 6.25 & $\mathrm{aC}$ \\
\hline LP 12-601 & 24.02 & a A & 20.15 & $\mathrm{aA}$ & 22.39 & $\mathrm{~b} \mathrm{~A}$ & 9.28 & $\mathrm{c} \mathrm{B}$ & 9.52 & $\mathrm{~b} \mathrm{~A}$ & 5.55 & $\mathrm{aB}$ \\
\hline BRS Valente & 23.05 & $\mathrm{aA}$ & 23.36 & $\mathrm{aA}$ & 21.61 & $\mathrm{~b} \mathrm{~A}$ & 8.33 & $\mathrm{c} \mathrm{A}$ & 9.06 & $\mathrm{~b} \mathrm{~A}$ & 5.75 & $\mathrm{aB}$ \\
\hline LP 11-363 & 23.29 & a B & 20.66 & $\mathrm{aB}$ & 26.15 & a A & 8.77 & c A & 8.65 & b A & 5.84 & $\mathrm{aB}$ \\
\hline CHP 99.6524 & 24.62 & a A & 22.41 & a A & 23.51 & $\mathrm{~b}$ A & 11.28 & $\mathrm{aA}$ & 10.47 & a A & 5.85 & a B \\
\hline LEP 04-14 & 27.33 & a A & 21.88 & a B & 27.70 & $\mathrm{aA}$ & 9.70 & c A & 10.25 & a A & 6.88 & a B \\
\hline G. Brilhante & 24.87 & $\mathrm{a} \mathrm{A}$ & 24.30 & $\mathrm{aA}$ & 24.31 & $\mathrm{aA}$ & 10.04 & b B & 11.33 & $\mathrm{aA}$ & 5.92 & $\mathrm{aC}$ \\
\hline CNFC 11954 & 18.99 & b B & 20.97 & $\mathrm{aB}$ & 24.53 & a A & 9.78 & c A & 8.42 & b B & 5.91 & $\mathrm{aC}$ \\
\hline CNFC 11948 & 19.81 & b B & 24.14 & a A & 25.35 & $\mathrm{a} \mathrm{A}$ & 11.36 & $\mathrm{aA}$ & 10.71 & $\mathrm{aA}$ & 6.17 & a B \\
\hline C10-2-4/41 & 24.75 & a A & 21.65 & a A & 24.41 & $\mathrm{a} \mathrm{A}$ & 9.26 & $\mathrm{c} \mathrm{A}$ & 10.14 & $\mathrm{aA}$ & 6.05 & $\mathrm{aB}$ \\
\hline Carioca & 24.30 & a A & 23.81 & a A & 22.33 & b A & 7.85 & c A & 8.63 & b A & 4.56 & a B \\
\hline LEC 03-14 & 24.92 & a A & 21.24 & a B & 25.72 & a A & 8.86 & c B & 10.42 & a A & 6.51 & $\mathrm{a} \mathrm{C}$ \\
\hline Mean & 22.88 & & 22.01 & & 23.98 & & 9.35 & & 9.78 & & 5.94 & \\
\hline VC (\%) & 8.22 & & 8.87 & & 6.91 & & 6.91 & & 6.79 & & 11.49 & \\
\hline
\end{tabular}

* Means not followed by the same lower case letter on a column differ by the Scott-Knott test $(\mathrm{P}=0.05)$, and the uppercase letters on the row differ by the Scott-Knott test $(\mathrm{P}=0.05) .{ }^{1}$ Environments: Env.1: 2014 rainy season; Env. 2: 2015 dry season; Env. 3: 2015 rainy season.

The LEC 03-14 line and the Carioca cultivar presented the highest calcium values in the three growing environments. These genotypes are promising for use in the calcium biofortification program. This mineral is essential for health as it participates in the biological function of various tissues, such as the musculoskeletal, nervous, and cardiac systems, and in bones and teeth (Martínez-Ballesta et al., 2010), and is therefore important for the prevention of osteoporosis.

Iron concentration in grains ranged from 63.00 to $140.68 \mathrm{mg} / \mathrm{kg}$ DM for the C10-24/41 (2015 dry season) and CHC 01-175-1 (2014 rainy season) lines, respectively. This range is similar to that described previously for common bean genotypes grown in Brazil (Silva et al., 2012; Jost et al., 2013; Ribeiro et al., 2013a,b). However, this is greater than the range observed for iron in common bean genotypes grown in other countries (Gelin et al., 2007; Nchimbi-Msolla and Tryphone, 2010; Pinheiro et al., 2010; Akond et al., 2011).

Genetics and Molecular Research 16 (1): gmr16019527 
The ranking of common bean genotypes with the highest iron concentration varied by growth environment. In the 2014 rainy season, the CHC 01-175-1, CHP 99.6524 , and LEP 0414 lines stood out above all cultivars evaluated. In the 2015 dry season, the CNFC 11948 line and the Guapo Brilhante cultivar had the highest iron values. Those two genotypes were also grouped by the Scott-Knott test in the group of genotypes superior for iron in the 2015 rainy season, without significantly differing from the LP 11-363, CHP 99.65 24, LEP 04-14, CNFC 11 954, C10-2-4/41, and LEC 03-14 lines. Of those lines, only CNFC 11948 showed an iron concentration superior to $95 \mathrm{mg} / \mathrm{kg} \mathrm{DM}$ in the three growing environments. The development of common bean cultivars with an iron concentration in the grains greater than $95 \mathrm{mg} / \mathrm{kg} \mathrm{DM}$ has been the goal of biofortification programs (Jost et al., 2013; Ribeiro et al., 2013a,b). Use of the CNFC 11948 line in the diet can help to reduce symptoms associated with iron deficiency, such as pallor, fatigue, weakness, dizziness, and reduced workability, which are common in individuals with anemia (Lynch, 2003).

The common bean genotypes presented zinc concentrations in their grains from 18.77 to $27.70 \mathrm{mg} / \mathrm{kg}$ DM. Higher zinc values have been previously described for common bean genotypes (Blair et al., 2010; Pinheiro et al., 2010; Talukder et al., 2010; Silva et al., 2012; Pereira et al., 2011, 2014; Ribeiro et al., 2014a; Maziero et al., 2015; Morais et al., 2016). None of the common bean genotypes studied showed a high zinc concentration, i.e., greater than 31 $\mathrm{mg} / \mathrm{kg}$ DM, according to the classification proposed by Tryphone and Nchimbi-Msolla (2010). Therefore, no common bean genotype is selected for use in the zinc biofortification program.

However, the identification of common bean genotypes containing low zinc concentration is also important for breeding programs, because some individuals need to reduce their dietary zinc intake. This is justified when it is necessary to normalize the levels of high density lipoprotein (HDL) in the blood and immune activity (Guerrero-Romero and Rodríguez-Morán, 2005). The selection of common bean lines with a low zinc concentration in their grains is a recent development in breeding programs and has shown promising results in the Andean gene pool (Zemolin et al., 2016).

The copper concentration in grains, as observed for other minerals, varied among genotypes and growing environments, from 4.56 (Carioca, 2015 rainy season) to $11.40 \mathrm{mg} / \mathrm{kg}$ DM (CHC 01-175-1, 2015 dry season). This range is similar to that observed in other studies in common bean genotypes (Pinheiro et al., 2010; Silva et al., 2012; Ribeiro et al., 2014a; Morais et al., 2016). However, the CHP 99.6524 and CNFC 11948 lines were grouped by the Skott-Knott test in the group of genotypes with the highest copper concentration in the three growing environments. Those lines are promising for use in copper biofortification programs. Increasing the copper concentration in common bean grains is desirable for the prevention of symptoms associated with copper deficiency, such as hypochromic anemia, neutropenia, and skeletal disorders (Guerrero-Romero and Rodríguez-Morán, 2005).

\section{Correlation between technological quality and biofortification traits}

Positive correlation estimates of moderate magnitude were obtained between phosphorus and potassium $(\mathrm{r}=0.575)$ and between iron and zinc $(\mathrm{r}=0.641)$ (Table 5). Low (Silva et al., 2012), moderate (Maziero et al., 2015), and high (Hossain et al., 2013) correlations have been reported between phosphorus and potassium in common bean grains. Correlation estimates described between iron and zinc in common bean grains range from moderate (Nchimbi-Msolla and Tryphone, 2010; Silva et al., 2012; Pereira et al., 2014; Morais

Genetics and Molecular Research 16 (1): gmr16019527 
et al., 2016) to high magnitude (Hossain et al., 2013). When the correlation estimates between two minerals are of moderate magnitude, the selection of one mineral will result in a moderate increase in the concentration of the other.

Table 5. Pearson correlation estimates between the traits mass of 100 grains (M100G), cooking time (CT), concentrations of potassium $(\mathrm{K})$, phosphorus $(\mathrm{P})$, calcium $(\mathrm{Ca})$, iron $(\mathrm{Fe})$, zinc $(\mathrm{Zn})$ and copper $(\mathrm{Cu})$ in grains of 14 common bean genotypes evaluated in three experiments carried out in 2014 and 2015.

\begin{tabular}{l|c|c|c|c|c|c|c}
\hline & $\mathrm{CT}$ & $\mathrm{K}$ & $\mathrm{P}$ & $\mathrm{Ca}$ & $\mathrm{Fe}$ & $\mathrm{Zn}$ & $\mathrm{Cu}$ \\
\hline $\mathrm{M} 100 \mathrm{G}$ & $0.122^{\mathrm{ns}}$ & $-0.157^{\mathrm{ns}}$ & $-0.168^{\mathrm{ns}}$ & $-0.280^{\mathrm{ns}}$ & $0.012^{\mathrm{ns}}$ & $-0.074^{\mathrm{ns}}$ & $0.071^{\mathrm{ns}}$ \\
\hline $\mathrm{CT}$ & & $0.150^{\mathrm{ns}}$ & $-0.301^{\mathrm{ns}}$ & $0.245^{\text {ns }}$ & $-0.245^{\text {ns }}$ & $-0.394^{\text {ns }}$ & $0.007^{\mathrm{ns}}$ \\
\hline $\mathrm{K}$ & & & $0.575^{*}$ & $-0.123^{\text {ns }}$ & $0.233^{\text {ns }}$ & $0.227^{\text {ns }}$ & $0.393^{*}$ \\
\hline $\mathrm{P}$ & & & & $-0.184^{\text {ns }}$ & $0.496^{\text {ns }}$ & $0.291^{\text {ns }}$ & $0.533^{*}$ \\
\hline $\mathrm{Ca}$ & & & & & $0.160^{\text {ns }}$ & $0.028^{\text {ns }}$ & $-0.100^{\text {ns }}$ \\
\hline $\mathrm{Fe}$ & & & & & & $0.641^{*}$ & $0.729^{*}$ \\
\hline $\mathrm{Zn}$ & & & & & & & $0.355^{\text {ns }}$ \\
\hline
\end{tabular}

*Significant by the Student's $t$-test $(\mathrm{P}=0.05)$; ns = non-significant.

Copper was highly positively correlated with iron $(\mathrm{r}=0.729)$ and moderately correlated with phosphorus $(\mathrm{r}=0.533)$. Previous studies have revealed no correlation between copper and iron in common bean grains (Silva et al., 2012; Hossain et al., 2013; Ribeiro et al., 2013b) or a correlation of low magnitude (Morais et al., 2016). Copper and phosphorus were also moderately correlated $(\mathrm{r}=0.630)$ in common bean genotypes evaluated by Hossain et al. (2013).

The study of correlations between minerals in common bean is of great importance for the development of biofortified cultivars. In the present study, an increase in phosphorus concentration was found to be accompanied by increases in potassium and copper concentrations, and increases in iron provided increments in the concentrations of zinc and copper in grains. This enables the development of common bean cultivars that are more nutritionally complete. Furthermore, the increased concentrations of potassium, phosphorus, calcium, iron, zinc, and copper did not result in an increase in cooking time of common bean grains, since these traits are not correlated. Similar results were observed in a preliminarily study by Ribeiro et al. (2013b) and Morais et al. (2016) in common bean genotypes. This indicates that there will be no change in the preparation time of biofortified common bean.

\section{Cluster analysis for technological quality and biofortification traits}

The first two principal components explained $99.9499 \%$ of the total variation (Table 6). The first principal component accumulated the highest percentage of total variation (98.6971\%). In this way, it was possible to identify the characteristics contributing the most to the genetic dissimilarity between genotypes evaluated: cooking time (0.9996), followed by the calcium (0.0012) and potassium (0.0007) concentrations. Several studies have shown genetic variability for the cooking time of common bean (Ribeiro et al., 2013a,b, 2014b; dos Santos et al., 2016; Morais et al., 2016). However, the present study is the first report showing that the cooking time of grains is an efficient descriptor to distinguish common bean genotypes. Regarding calcium, Pereira et al. (2011) observed that this mineral had the greatest and lowest contribution to the genetic dissimilarity of common bean genotypes in the first and second year of growth, respectively. Therefore, calcium is a descriptor that may or may not be efficient in the differentiation of germplasm.

Genetics and Molecular Research 16 (1): gmr16019527 
Table 6. Estimation of eigenvalues (root and \% cumulative) and relative importance of the characters mass of 100 grains (M100G), cooking time (CT), concentrations of potassium $(\mathrm{K})$, phosphorus $(\mathrm{P})$, calcium $(\mathrm{Ca})$, iron $(\mathrm{Fe})$, zinc $(\mathrm{Zn})$, and copper $(\mathrm{Cu})$ in the grains obtained in each principal component $(\mathrm{PC})$ to estimate the genetic dissimilarity among 14 common bean genotypes evaluated in three experiments carried out in 2014 and 2015 .

\begin{tabular}{|c|c|c|c|c|c|c|c|c|c|c|}
\hline \multirow[t]{2}{*}{$\mathrm{PC}$} & \multirow[t]{2}{*}{$\operatorname{Root}(\%)$} & \multirow[t]{2}{*}{ Cumulative (\%) } & \multirow[t]{2}{*}{ M100G (g) } & \multirow[t]{2}{*}{ CT (s) } & $\mathrm{K}$ & $\mathrm{P}$ & $\mathrm{Ca}$ & $\mathrm{Fe}$ & $\mathrm{Zn}$ & $\mathrm{Cu}$ \\
\hline & & & & & \multicolumn{3}{|c|}{$\mathrm{g} / \mathrm{kg}$ dry matter } & \multicolumn{3}{|c|}{$\mathrm{mg} / \mathrm{kg}$ dry matter } \\
\hline PC 1 & 98.6971 & 98.6971 & 0.0021 & 0.9996 & 0.0007 & -0.0010 & 0.0012 & -0.0289 & -0.0062 & 0.0000 \\
\hline PC 2 & 1.2528 & 99.9499 & 0.0069 & 0.0292 & 0.0112 & 0.0122 & 0.0099 & 0.9947 & 0.0791 & 0.0554 \\
\hline PC 3 & 0.0302 & 99.9801 & 0.9900 & -0.0025 & -0.0516 & -0.0263 & -0.0954 & 0.0003 & -0.0824 & 0.0238 \\
\hline PC 4 & 0.0129 & 99.9930 & 0.0847 & 0.0038 & 0.0618 & -0.0346 & -0.0177 & -0.0748 & 0.9876 & -0.0806 \\
\hline PC 5 & 0.0041 & 99.9971 & -0.0338 & -0.0005 & 0.4123 & 0.2460 & -0.4885 & -0.0456 & 0.0327 & 0.7256 \\
\hline PC 6 & 0.0014 & 99.9986 & 0.0426 & 0.0002 & 0.7300 & 0.3133 & -0.1118 & 0.0284 & -0.0862 & -0.5886 \\
\hline PC 7 & 0.0011 & 99.9997 & 0.0974 & -0.0023 & 0.3431 & 0.1373 & 0.8590 & -0.0348 & 0.0154 & 0.3385 \\
\hline PC 8 & 0.0003 & 100.0000 & 0.0115 & 0.0012 & -0.4157 & 0.9058 & 0.0375 & -0.0084 & 0.0529 & -0.0479 \\
\hline
\end{tabular}

The first two principal components separated the common bean genotypes into four groups - $\mathrm{G}$ (Figure 1). Among the groups, there is genetic dissimilarity, especially for cooking time (G3), calcium concentration (G2), and iron concentration (G1). The LP 11-363 line (G3) has the lowest cooking time and should be used in controlled crossings with the CNFC 11 948 line (G2), which has a high iron concentration. The controlled crossing of the LP 11-363 line (G3) is also indicated with the LEC 03-14 line, which has a high potassium, phosphorus, and calcium concentration. Recombinants with fast-cooking time that are biofortified with minerals can be selected from the segregating generations obtained. The obtained progenies should be evaluated for phytate concentration, because phytates have an inhibitory effect on iron and zinc absorption by the body (Ariza-Nieto et al., 2007).

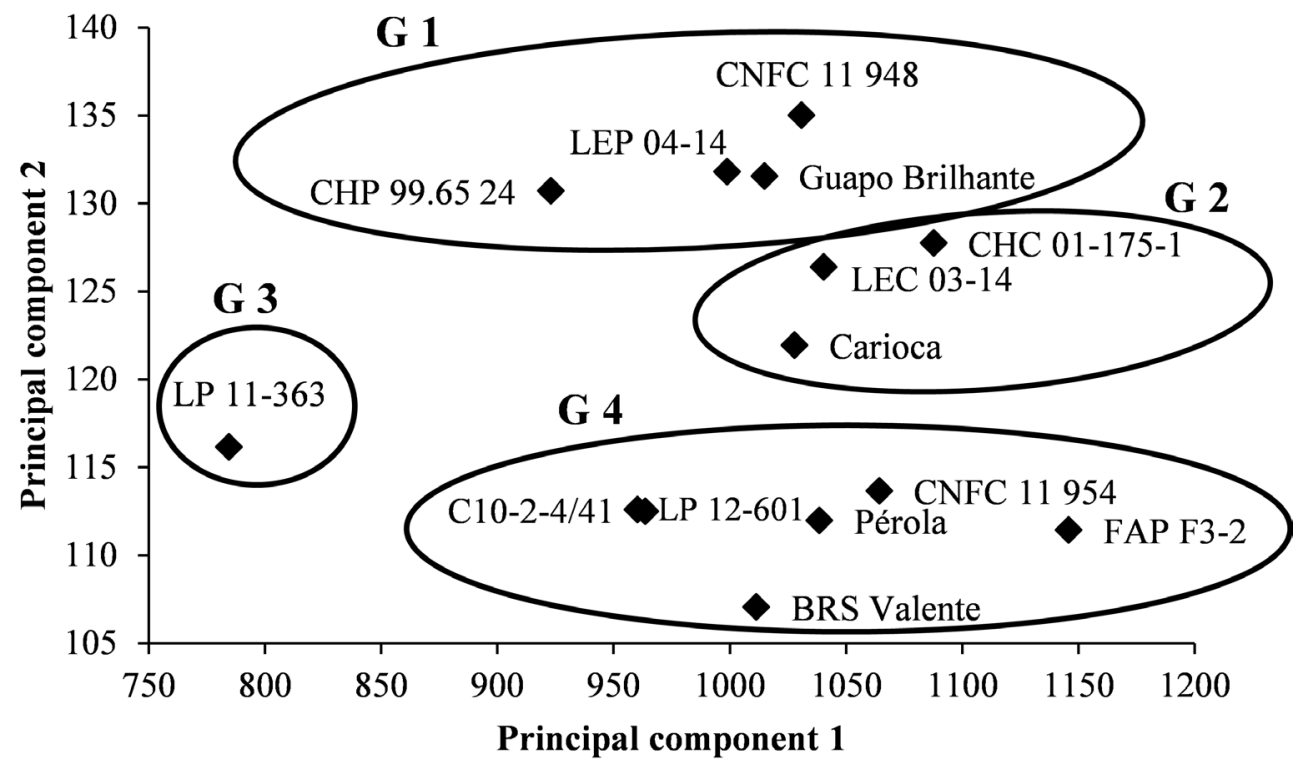

Figure 1. Dispersion graph obtained from the technological quality descriptors and biofortification of 14 common bean genotypes for the first two main components (PC1 and PC2).

Genetics and Molecular Research 16 (1): gmr16019527 
The development of common bean cultivars biofortified with minerals by genetic breeding represents a technological advance and offers food with higher nutritional value that can contribute to the prevention of mineral deficiencies in humans. The biofortification of beans with minerals no increase the cooking time and the costs with the preparation of food, and meets the approach of food security.

\section{Conflicts of interest}

The authors declare no conflict of interest.

\section{ACKNOWLEDGMENTS}

We would like to thank Conselho Nacional de Desenvolvimento Científico e Tecnológico $(\mathrm{CNPq})$ for providing financial aid and granting scholarships. We would also like to thank Coordenação de Aperfeiçoamento de Pessoal de Nível Superior (Capes) and Fundação de Amparo à Pesquisa do Estado de Minas Gerais (FAPEMIG) for the grants awarded. The authors would also like to thank Dra. Sandra Maria Maziero for his assistance performing the cluster analysis.

\section{REFERENCES}

Akond ASMGM, Crawford H, Berthold J, Talukder ZI, et al. (2011). Minerals (Zn, Fe, Ca and Mg) and antinutrient (phytic acid) constituents in common bean. Am. J. Food Tech 6: 235-243. http://dx.doi.org/10.3923/ajft.2011.235.243

Ariza-Nieto M, Blair MW, Welch RM and Glahn RP (2007). Screening of iron bioavailability patterns in eight bean (Phaseolus vulgaris L.) genotypes using the Caco-2 cell in vitro model. J. Agric. Food Chem. 55: 7950-7956. http:// dx.doi.org/10.1021/jf070023y

Blair MW, González LF, Kimani PM and Butare L (2010). Genetic diversity, inter-gene pool introgression and nutritional quality of common beans (Phaseolus vulgaris L.) from Central Africa. Theor. Appl. Genet. 121: 237-248. http:// dx.doi.org/10.1007/s00122-010-1305-x

Carbonell SAM, Chiorato AF, Gonçalves JGR, Perina EF, et al. (2010). Tamanho de grão comercial em cultivares de feijoeiro. Cienc. Rural 40: 2067-2073. http://dx.doi.org/10.1590/S0103-84782010005000159

Cruz CD (2016). Genes software - extended and integrated with the R, Matlab and Selegen. Acta Sci. Agron. 38: $547-552$. http://dx.doi.org/10.4025/actasciagron.v38i3.32629

Cruz CD, Regazzi AJ and Carneiro PCS (2012). Modelos biométricos aplicados ao melhoramento genético, 4nd edn. Universidade Federal de Viçosa, Viçosa.

dos Santos GG, Ribeiro ND and Maziero SM (2016). Evaluation of common bean morphological traits identifies grain thickness directly correlated with cooking time. Pesqui. Agropecu. Trop. 46: 35-42. http://dx.doi.org/10.1590/1983$\underline{40632016 \mathrm{v} 4638191}$

Gelin JR, Forster S, Grafton KF, McClean PE, et al. (2007). Analysis of seed zinc and other minerals in a recombinant inbred population of navy bean (Phaseolus vulgaris L.). Crop Sci. 47: 1361-1366. http://dx.doi.org/10.2135/ cropsci2006.08.0510

Guerrero-Romero F and Rodríguez-Morán M (2005). Complementary therapies for diabetes: the case for chromium, magnesium, and antioxidants. Arch. Med. Res. 36: 250-257. http://dx.doi.org/10.1016/j.arcmed.2005.01.004

Hacisalihoglu G and Settles AM (2013). Natural variation in seed composition of 91 common bean genotypes and their possible association with seed coat color. J. Plant Nutr. 36: 772-780. http://dx.doi.org/10.1080/01904167.2012.754041

He FJ and MacGregor GA (2008). Beneficial effects of potassium on human health. Physiol. Plant. 133: 725-735. http:// dx.doi.org/10.1111/j.1399-3054.2007.01033.x

Hossain KG, Islam N, Jacob D, Ghavami F, et al. (2013). Interdependence of genotype and growing site on seed mineral compositions in common bean. Asian J. Plant Sci. 12: 11-20. http://dx.doi.org/10.3923/ajps.2013.11.20

Jost E, Ribeiro ND, Maziero SM, Possobom MTDF, et al. (2013). Comparison among direct, indirect and index selections on agronomic traits and nutritional quality traits in common bean. J. Sci. Food Agric. 93: 1097-1104. http://dx.doi. org/10.1002/jsfa.5856

Genetics and Molecular Research 16 (1): gmr16019527 
Lynch SR (2003). Iron deficiency anemia. In: Encyclopedia of food sciences and nutrition. Academic Press, Amsterdam, 215-320.

Martínez-Ballesta MC, Dominguez-Perles R, Moreno DA, Muries B, et al. (2010). Minerals in plant food: effect of agricultural practices and role in human health. A review. Agron. Sustain. Dev. 30: 295-309. http://dx.doi.org/10.1051/ agro/2009022

Maziero SM, Ribeiro ND and Storck L (2015). Simultaneous selection in beans for architecture, grain yield and minerals concentration. Euphytica 205: 369-380. http://dx.doi.org/10.1007/s10681-015-1392-5

Montgomery DC and Peck EA (1981). Introduction to linear regression analysis. 1st edn. J Wiley, New York.

Morais NM, Ribeiro ND, Storck L, dos Santos PRF, et al. (2016). Selection of common bean land cultivars based on agronomic performance, cooking time, and mineral concentration. Semin. Cienc. Agrar. 37: 1255-1266. http://dx.doi. org/10.5433/1679-0359.2016v37n3p1255

Nchimbi-Msolla S and Tryphone GM (2010). The effects of the environment on iron and zinc concentrations and performance of common bean (Phaseolus vulgaris L.) genotypes. Asian J. Plant Sci. 9: 455-462. http://dx.doi. org/10.3923/ajps.2010.455.462

Pereira HS, Del Peloso MJ, Bassinello PZ, Guimarães CM, et al. (2014). Genetic variability for iron and zinc content in common bean lines and interaction with water availability. Genet. Mol. Res. 13: 6773-6785. http://dx.doi. org/10.4238/2014.August.28.21

Pereira T, Coelho CMM, dos Santos JCP, Bogo A, et al. (2011). Diversidade no teor de nutrientes em grãos de feijão crioulo no Estado de Santa Catarina. Acta Sci. Agron. 33: 477-485.

Pinheiro C, Baeta JP, Pereira AM, Domingues H, et al. (2010). Diversity of seed mineral composition of Phaseolus vulgaris L. germplasm. J. Food Compos. Anal. 23: 319-325. http://dx.doi.org/10.1016/j.jfca.2010.01.005

Prasad R (2012). Micro mineral nutrient deficiencies in humans, animals and plants and their amelioration. Proc. Natl. Acad. Sci. USA 82: 225-233

Ribeiro ND, Cargnelutti Filho A, Poersch NL and Rosa SS (2007). Padronização de metodologia para avaliação do tempo de cozimento dos grãos de feijão. Bragantia 66: 335-348. http://dx.doi.org/10.1590/S0006-87052007000200018

Ribeiro ND, Domingues L da S, Zemolin AEM and Possobom MTDF (2013a). Selection of common bean lines with high agronomic performance and high calcium and iron concentrations. Pesqui. Agropecu. Bras. 48: 1368-1375. http:// dx.doi.org/10.1590/S0100-204X2013001000008

Ribeiro ND, Mambrin RB, Storck L, Prigol M, et al. (2013b). Combined selection for grain yield, cooking quality and minerals in the common bean. Rev. Cienc. Agron 44: 869-877. http://dx.doi.org/10.1590/S1806-66902013000400025

Ribeiro ND, Rodrigues J de A, Prigol M, Nogueira CW, et al. (2014a). Evaluation of special grains bean lines for grain yield, cooking time and mineral concentration. Crop Breed. Appl. Biotechnol. 14: 15-22. http://dx.doi.org/10.1590/ $\underline{\text { S1984-70332014000100003 }}$

Ribeiro ND, Domingues L da S, Gruhn EM, Zemolin AEM, et al. (2014b). Desempenho agronômico e qualidade de cozimento de linhagens de feijão de grãos especiais. Rev. Cienc. Agron. 45: 92-100. http://dx.doi.org/10.1590/S180666902014000100012

Silva CA, Abreu AFB, Ramalho MAP and Maia LGS (2012). Chemical composition as related to seed color of common bean. Crop Breed. Appl. Biotechnol. 12: 132-137. http://dx.doi.org/10.1590/S1984-70332012000200006

Talukder ZI, Anderson E, Miklas PN, Blair MW, et al. (2010). Genetic diversity and selection of genotypes to enhance Zn and Fe content in common bean. Can. J. Plant Sci. 90: 49-60. http://dx.doi.org/10.4141/CJPS09096

Tryphone GM and Nchimbi-Msolla S (2010). Diversity of common bean (Phaseolus vulgaris L.) genotypes in iron and zinc contents under screenhouse conditions. J. Agric. Res. 5: 738-747.

Zemolin AEM, Ribeiro ND, Casagrande CR, da Silva MJ, et al. (2016). Genetic parameters of iron and zinc concentrations in Andean common bean seeds. Acta Sci. Agron. 38: 439-446. http://dx.doi.org/10.4025/actasciagron.v38i4.30652

Genetics and Molecular Research 16 (1): gmr16019527 